This accepted author manuscript is copyrighted and published by Elsevier. It is posted here by agreement between Elsevier and MTA. The definitive version of the text was subsequently published in [Hormones and Behavior, Vol. 66., Issue 3., August 2014, DOI 10.1016/j.yhbeh.2014.08.002]. Available under license CC-BY-NC-ND. 
Regular article

\title{
The effects of lactation on impulsive behavior in vasopressin-deficient Brattleboro rats
}

\author{
Mano Aliczki a,*, Anna Fodor ${ }^{\mathrm{a}, \mathrm{b}}$, Zoltan Balogh a, Jozsef Haller ${ }^{\mathrm{a}}$, Dora Zelena ${ }^{\mathrm{a}}$ \\ a Department of Behavioural Neurobiology_Institute of Experimental Medicine, Hungarian Academy of Science, Budapest, Hungary \\ b Janos Szentagothai School of Neuroscien S, nmelweis University, Budapest, Hungary
}

\section{A R T I C L E I N F O}

\section{Article history:}

Received 24 December 2013

Revised 31 July 2014

Accepted 2 August 2014

Available online $\mathrm{xxxx}$

\section{Keywords:}

Brattleboro

Chlordiazepoxide

Corticosterone

Imipramine

Impulsivity

Lactation

Rat

Vasopressin

\begin{abstract}
A B S T R A C T
Vasopressin (AVP)-deficient Brattleboro rats develop a specific behavioral profile, which-among other things- 22 include altered cognitive performance. This profile is markedly affected by alterations in neuroendocrine state 23 of the animal such as during lactation. Given the links between AVP and cognition we hypothesized that AVP de- 24 ficiency may lead to changes in impulsivity that is under cognitive control and the changes might be altered by 25 lactation. Comparing virgin and lactating AVP-deficient female Brattleboro rats to their respective controls, we 26 assessed the putative lactation-dependent effects of AVP deficiency on impulsivity in the delay discounting par- 27 adigm. Furthermore, to investigate the basis of such effects, we assessed possible interactions of AVP deficiency 28 with GABAergic and serotonergic signaling and stress axis activity, systems playing important roles in impulse 29 control. Our results showed that impulsivity was unaltered by AVP deficiency in virgin rats. In contrast a 30 lactation-induced increase in impulsivity was abolished by AVP deficiency in lactating females. We also found 31 that chlordiazepoxide-induced facilitation of GABAergic and imipramine-induced enhancement of serotonergic 32 activity in virgins led to increased and decreased impulsivity, respectively. In contrast, during lactation these ef- 33 fects were visible only in AVP-deficient rats. These rats also exhibited increased stress axis activity compared to 34 virgin animals, an effect that was abolished by AVP deficiency. Taken together, AVP appears to play a role in the 35 regulation of impulsivity exclusively during lactation: it has an impulsivity increasing effect which is potentially 36 mediated via stress axis-dependent mechanisms and fine-tuning of GABAergic and serotonergic function.
\end{abstract}

(C) 2014 Published by Elsevier Inc.

\section{Introduction}

Arginine-vasopressin (AVP) is a peptide hormone produced in the supraoptic and paraventricular nuclei of the hypothalamus (Rhodes et al., 1981; Sokol et al., 1976). Its primary physiological function is to stimulate water retention by increasing the water permeability of the distal tubules of the kidneys (Flamion and Spring, 1990; Wade et al., 1981). However, AVP also acts at vasopressin receptors at several brain areas (Buijs et al., 1978) to regulate a number of neuroendocrine and behavioral processes (Antoni, 1993).

The Brattleboro homozygous recessive rat does not synthesize AVP (Bohus and de Wied, 1998) and is thus a useful model for studying the role of AVP in behavioral processes. Brattleboro rats develop a unique physiological and behavioral profile as a result of lacking a functioning AVP system. Among other things, these rats show normal baseline hypothalamus-pituitary-adrenal (HPA) axis activity and decreased HPA axis reactivity to a variety of stressors (Zelena et al., 2009), slightly reduced anxiety (Fodor et al., 2012) and depression-like behavior

\footnotetext{
* Corresponding author at: Institute of Experimental Medicine, P.O. Box 67, 1450 Budapest, Hungary. Fax: + 3612109951.

E-mail address: aliczki.mano@koki.mta.hu (M. Aliczki).
}

(Fodor et al., 2012; Mlynarik et al., 2007). Additionally, they display so- 60 cial deficits (Engelmann and Landgraf, 1994; Feifel et al., 2009; Schank, 61 2009) and impairments in cognitive performance (Aarde and Jentsch, 62 2006; Colombo et al., 1992; Varga et al., 2013). The behavioral effects 63 of AVP deficiency are thought to depend on the neuroendocrine state 64 of the individual, e.g. in several cases on the specific physiological con- 65 ditions during lactation. For example, AVP deficiency does not alter 66 baseline HPA axis activity in virgin females, while it dampens chronic 67 hyperactivity of the HPA axis in lactating female rats (Fodor et al., 68 2013), an effect that contributes to maternal neglect and mild anxiolysis 69 (Fodor et al., 2012).

Prior work has shown that cognitive performance can be altered by 71 changes in impulsivity (Bizot and Thiebot, 1996). Impulsivity is general- 72 ly characterized by a failure to resist a drive to respond to environmen- 73 tal stimuli (motor impulsivity) and by responses without consideration 74 of alternatives and/or future consequences (choice impulsivity) 75 (Evenden and Ryan, 1996; Kim and Lee, 2011; Solanto et al., 2001). 76 While it is possible that impulsivity impacts cognitive performance, it 77 is also probable that cognitive and various physiological processes affect 78 impulsivity (Aron, 2007). Thus, as cognitive functions are altered in 79 AVP-deficient rats, one might assume that impulsive behavior is also 80 affected. 
In the present study, firstly we aimed to identify the effects of AVP deficiency on impulsive behavior. As AVP deficiency can alter behavioral processes in a lactation-dependent manner, we also studied possible interactions between AVP activity and lactation in the regulation of impulsivity using virgin and lactating female AVP-deficient and control Brattleboro rats. Specifically, we used the delay discounting paradigm to study impulsive behavior. In this paradigm preference of a delayed, large reward over a smaller, immediate reward is tested with the employment of an operant conditioning procedure. Typically, impulsive individuals tend to choose the latter type of reinforcer in similar paradigms (Adriani and Laviola, 2003; Adriani et al., 2003b; Bizot et al., 1999; Evenden and Ryan, 1996, 1999; Thiebot et al., 1985). Prior to investigations of impulsive behavior, we also assessed cognitive performance of AVP-deficient rats during the training phase of the delay discounting paradigm.

Showing that AVP deficiency decreases impulsivity in lactating rats, our second aim was to assess the basis of such effects. GABAergic and serotonergic signaling play important roles in the regulation of impulsive behavior; pharmacological manipulation of these systems leads to changes in impulsivity (Bizot et al., 1999; Evenden and Ko, 2005). As AVP activity was shown to alter both GABAergic and serotonergic function (Auerbach and Lipton, 1982; Hermes et al., 2000; Ramanathan et al., 2012; Schwarzberg et al., 1981; Wang et al., 2002), we studied whether AVP deficiency exerts its impulsivity altering effects via possible GABAergic and serotonergic interactions. To assess such interactions, we investigated impulsive behavior in AVP-deficient virgin and lactating female Brattleboro rats following treatment with a benzodiazepine, which has been reported to increase impulsivity (Evenden and Ko, 2005; Thiebot et al., 1985; Wolff and Leander, 2002), or a selective serotonin reuptake inhibitor, which has been reported to decrease impulsivity in several studies (Bizot et al., 1988; Miyazaki et al., 2011). In addition to measurements of impulsive behavior, HPA axis activity (i.e. corticosterone levels) was also assessed, as the HPA axis has been reported to be altered by AVP deficiency (Fodor et al., 2013; Makara et al., 2012) and to play a role in impulsivity (Torregrossa et al., 2012).

\section{Material and methods}

\section{Subjects}

We compared AVP-deficient homozygous female rats with homozygous control $(+/+)$ rats. AVP-deficient and control Brattleboro rats came from a colony maintained in our Institute. The breeding stock was started from breeder rats provided by Harlan Laboratories (Indianapolis, USA). The parents of control rats were homozygous for the non-mutated gene, while AVP-deficient subjects originated from breeding pairs composed of AVP-deficient fathers and heterozygous mothers. Heterozygous mothers always derived from control and AVP-deficient parents, to keep the genetic background of the two lines close. Animals were kept on a light/dark cycle of $12 \mathrm{~h}$ with the lights on at $0700 \mathrm{~h}$. The temperature and humidity were kept at $23 \pm 2{ }^{\circ} \mathrm{C}$ and $60 \pm 10 \%$, respectively. Virgin female rats were isolated one week before the start of experimentation and housed individually until the end of all experiments. Female rats that were studied during lactation were mated at the age of 75-115 days and were isolated approximately one week before delivery. Female subjects mated with males of different homozygous genotype, i.e. AVP-deficient females mated with control males, while control females mated with AVP-deficient males. With this design the genotype of all pups was heterozygous; therefore, litter genotype did not differ between subjects and it could not alter maternal behavior. Virgin and lactating females were the same age at the time of experimentation. One day after delivery litters were culled to three males and three females to control for the behavioral effects of quantity and quality of pups. Pups were housed with their dam throughout the experiments (except for during experimentation in the delay discounting boxes). Tap water was available ad libitum. Rat chow was limited to 6 pellets a day (approximately $20 \mathrm{~g}$ total) to in- 145 crease exploration during the delay discounting experiments. Food 146 was provided immediately after the daily training/testing sessions. 147 The weight of each rat was measured daily. Food restriction was adjust- 148 ed where necessary to maintain the rats at a minimum of $80 \%$ of their 149 starting weight. Pups were also evaluated daily to monitor their devel- 150 opment. All animals survived experimentation and showed no sign of 151 pain or discomfort throughout our studies. All experiments were con- 152 ducted in accordance with the European Communities Council Directive 153 of 24 November 1986 (86/609/EEC) and were reviewed and approved 154 by the Animal Welfare Committee of the Institute of Experimental 155 Medicine, Budapest, Hungary.

Drugs and doses

The benzodiazepine, chlordiazepoxide (CDP), and the tryciclic anti- 158 depressant, imipramine (IMI), were dissolved in saline. These drugs 159 were administered intraperitoneally 15 min (CDP) or 60 min (IMI) be- 160 fore the start of the experiment at a dose of 0 (vehicle) or $10 \mathrm{mg} / \mathrm{kg}$ in a 161 volume of $1 \mathrm{ml} / \mathrm{kg}$. The doses, volume, injection routes and pretreat- 162 ment time were determined based on previous studies (Evenden and 163 Ko, 2005; Evenden, 1998).

Experiments assessing impulsive behavior were conducted using 166 automated operant chambers equipped with two nose-poke holes 167 with infrared sensors and LED lights, a chamber light and a feeder device 168 with a magazine into which food pellets were dropped (Med Associates, 169 St. Albans, VT, USA). Chambers were placed inside sound-attenuated 170 wooden cubicles and were controlled via computers running Med-PC 171 IV software (Med Associates, St. Albans, VT, USA).

During the training phase, animals were placed inside a chamber for 173 30 min daily for 5 days. A response on one of the nose-poke holes was 174 rewarded with one $45 \mathrm{mg}$ food pellet (small reward), while a response 175 on the other hole resulted in five $45 \mathrm{mg}$ food pellets (large reward). 176 Both types of reward were presented immediately after the response 177 and were followed by a $25 \mathrm{~s}$ timeout with the chamber light switched 178 on. Chamber light was used as a cue which could be associated with 179 the reward after responding on one of the nose-poke holes. It is a com- 180 mon practice to associate visual or auditory cues with the feedback to 181 accelerate learning in operant conditioning procedures (Panlilio et al., 182 2012). During the timeout period, responses were not rewarded but 183 were registered. To avoid side preference, the nose-poke hole on 184 which responding was rewarded with five food pellets was randomly 185 assigned to either the left or the right side between animals. Animals 186 were placed in the same chamber with the same nose-poke hole side as- 187 signment throughout the experiment. After each session ended, the 188 chambers were cleaned with 70\% ethanol and were dried with paper 189 towels. All experiments were conducted in the early hours of the light 190 phase. At the end of the training phase, the animals were expected to re- 191 spond on the nose-poke hole that was paired with the large reward in 192 approximately $90 \%$ of all trials (Adriani et al., 2003a).

After two days of rest, the animals underwent the test phase. During 194 this phase, each animal was placed in a chamber for 30 min daily for 195 8 days. The procedure was similar to that described for the training 196 phase, but a delay was inserted before the large reward. The delay 197 was fixed for each daily session and was increased progressively over 198 subsequent days (10, 20, 30, 45, 60, 80, 100 and 120 s). Responses dur- 199 ing these delays were not rewarded, but they were recorded by the soft- 200 ware. Sessions of the test phase were conducted at the same time as 201 sessions of the training phase. During the test phase, subjects were 202 expected to shift their preference from the nose-poke hole rewarded 203 by the delayed large reward to the nose-poke hole rewarded by the im- 204 mediate small reward (Adriani and Laviola, 2003; Adriani et al., 2003b). 205 
During the training sessions, we recorded the preference of the nose-poke hole paired with the large reward (large reward preference) to assess learning capabilities. Increases of greater magnitude in large reward preference indicated quicker learning. During the test phase, large reward preferences were indicative of non-impulsive choices. This variable is negatively associated with choice impulsivity, which refers to an inability to prefer a larger, delayed reward over an immediate smaller one (Kim and Lee, 2011). Because a slight difference in large reward preference was observed between the treatment groups on the last day of the training phase (see Results), large reward preference during the test phase was calculated as a percentage of the large reward preference on the last day of training (\%). With this method, we were able to assess the changes in large reward preference throughout the testing phase. The number of inadequate responses (the sum of responses during timeouts and delays), which reflects the number of premature, impulsive responses, was also evaluated. With this measure, we were able to assess motor impulsivity, which is defined as the inability to inhibit inappropriate actions (Kim and Lee, 2011).

\section{Blood sampling and hormone measurements}

For corticosterone measurements, trunk blood was collected in icecold plastic tubes following decapitation in the early hours of the light phase. After sampling, blood was centrifuged at $4{ }^{\circ} \mathrm{C}$, and the serum was separated and stored at $-20^{\circ} \mathrm{C}$ until analysis. Serum corticosterone was measured in $10 \mu \mathrm{l}$ unextracted serum by a radioimmunoassay (RIA) using a specific antibody developed in our institute as described earlier (Zelena et al., 2003). The corticosterone antibody was raised in rabbits against corticosterone-carboxymethyloxime bovine serum albumine. ${ }^{125}$ I-labeled corticosterone-carboxymethyloxime-tyrosine methyl ester was used as tracer. The interference from plasma transcortin was eliminated by inactivating transcortin at a low $\mathrm{pH}$. Assay sensitivity was $1 \mathrm{pmol}$. The intraassay coefficient of variation was $7.5 \%$. All the samples from a particular experiment were measured in one RIA.

\section{Experimental design}

Each experiment was performed on a separate set of animals and was analyzed separately. All experiments were carried out in the early hours of the light phase.

\section{Assessment of cognitive performance and impulsivity in virgin females}

In Experiment 1, we examined learning and impulsive behavior in virgin AVP-deficient $(N=10)$ and control female Brattleboro rats $(N=10)$. Subjects underwent 4 days of partial food restriction then underwent the training phase of the delay discounting paradigm (assessment of cognitive performance), throughout which they were food restricted. The last day of the training phase was followed by two days of rest then subjects underwent the test phase of the delay discounting procedure. Animals underwent three additional daily sessions after the last day of the test phase with 120 s delay. Before these sessions, each animal received either an injection of vehicle, $10 \mathrm{mg} / \mathrm{kg}$ $\mathrm{CDP}$ or $10 \mathrm{mg} / \mathrm{kg}$ IMI in a random order, with one treatment each day. The effects of CDP and IMI on impulsivity were compared to the effects of control (vehicle) treatment received each day.

\section{Assessment of cognitive performance and impulsivity in lactating females}

Experiment 2 was conducted in a similar manner as described for Experiment 1, except that the subjects were control $(N=10)$ and AVP-deficient $(N=10)$ lactating female rats that had delivered 1-5 days before the first day of the test phase. Food restriction was started immediately after delivery.
Assessment of HPA axis activity in virgin and lactating females

In Experiment 3, we assessed HPA axis activity in two separate sets of 263 virgin and lactating AVP-deficient and control female Brattleboro rats. 264 The first set of animals consisted of control $(N=12)$ and AVP- 265 deficient $(N=12)$ lactating rats from which blood was sampled 266 on the 10th day after delivery and control $(N=6)$ and AVP-deficient 267 $(N=8)$ virgin rats which were in the same age as lactating rats at the 268 time of blood sampling. This time point had been selected to coincide 269 with the lactation day at the end of the training phase of the delay 270 discounting test (see Experiments 1 and 2). For the second set of animals 271 blood sampling was carried out in a similar manner (experimental 272 groups were lactating control $(N=17)$, lactating AVP-deficient $(N=273$ $17)$, virgin control $(N=11)$ and virgin AVP-deficient $(N=16)$ ), except 274 blood was sampled from lactating rats on the 20th days following deliv- 275 ery. Blood was sampled from virgin rats which were at the same age 276 as lactating rats at the time of blood sampling.

Statistical analyses

Data were presented as the mean \pm standard error of the mean. Be- 279 havioral variables of the delay discounting test (large reward preference 280 and inadequate responses) were analyzed using repeated measures 281 analysis of variance (ANOVA) (training and test phases of Experiment 282 1 and 2: factor 1: genotype; repeated factor: days or delay; during 283 pharmacological treatments in Experiment 1 and 2: factor 1: genotype; 284 repeated factor: treatment). Corticosterone levels were analyzed using 285 factorial ANOVA (Experiment 3: factor 1: genotype; factor 2: lactation). 286 ANOVA assumptions were evaluated by Levene's test. Duncan tests 287 were performed for post-hoc analyses when a main effect was signifi- 288 cant, and Bonferroni corrections were applied for multiple comparisons. 289 $P$ values less than 0.05 were considered statistically significant.

Results

Cognitive performance and impulsivity in AVP-deficient and control virgin 292 females

Large reward preference was significantly increased throughout the 294 training phase in virgin females $\left(\mathrm{F}_{\text {days }}(4,72)=16.1 ; p<0.01\right)$. Rats 295 lacking AVP exhibited a reduced general preference for the large reward 296 compared to control animals $\left(\mathrm{F}_{\text {genotype }}(1,18)=7.37 ; p=0.01\right.$ ) (Fig. 1$) .297$ No significant interaction between days of the test phase and genotype 298 was observed $\left(\mathrm{F}_{\text {genotype }} \times\right.$ delay $\left.(4,72)=1.66 ; p=0.17\right) . \quad 299$

Delay significantly decreased large reward preference in virgin 300 female rats across days $\left(\mathrm{F}_{\text {delay }}(7,126)=13.25 ; p<0.01\right)$, but genotype 301 failed to alter this variable $\left(F_{\text {genotype }}(1,18)=1.35 ; p=0.26 ; 302\right.$ $\mathrm{F}_{\text {genotype } \times \text { delay }}(7,126)=1.69 ; p=0.12$ ) (Fig. 2a). The number of 303 inadequate responses was also affected by delay, as they increased 304 throughout the test phase $\left(\mathrm{F}_{\text {delay }}(7,126)=5.55 ; p<0.01\right)$, but it 305 was not altered by genotype $\left(\mathrm{F}_{\text {genotype }}(1,18)=1.09 ; p=0.31 ; 306\right.$ $\left.\mathrm{F}_{\text {genotype } \times \text { delay }}(7,126)=1.03 ; p=0.41\right)$ (Fig. $\left.2 b\right)$.

In virgin females, large reward preference was unaltered by 308 genotype, treatment or the interaction between these two factors 309 $\left(F_{\text {genotype }}(1,18)=1.06 ; p=0.32 ; F_{\text {treatment }}(2,36)=0.52 ; p=0.6 ; 310\right.$ $\left.\mathrm{F}_{\text {genotype } \times \text { treatment }}(2,36)=0.89 ; p=0.42\right)$ during the three-day peri- 311 od of pharmacological treatments (Fig. 2c). Inadequate responses were 312 unchanged by genotype $\left(\mathrm{F}_{\text {genotype }}(1,18)=0.3 ; p=0.59\right)$, but 313 they were significantly altered by treatment $\left(\mathrm{F}_{\text {treatment }}(2,36)=19.67 ; 314\right.$ $p<0.01$ ) (Fig. 2d). Post-hoc comparisons revealed that CDP increased 315 the number of inadequate responses, while IMI decreased inadequate 316 responding compared to vehicle treatment. The effects of these treat- 317 ments were independent of genotype $\left(F_{\text {genotype }} \times\right.$ treatment $(2,36)=318$ $1.37 ; p=0.26$ ). 


\section{Virgin females}

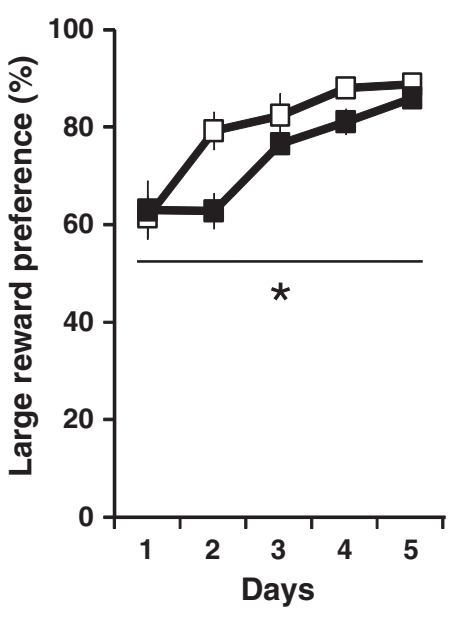

Control

AVP-deficient

Fig. 1. Effects of vasopressin (AVP) deficiency on learning in virgin female Brattleboro rats in the training phase of the delay discounting paradigm (Experiment 1). AVP-deficient rats showed an overall decreased learning ability. ${ }^{*}$ under the line denotes an overall significant difference between the two genotypes $(p<0.05)$.

\section{Cognitive performance and impulsivity in AVP-deficient and control lactat-} ing females

Large reward preference was significantly increased during the training phase in all lactating rats regardless of their genotype $\left(\mathrm{F}_{\text {days }}(4,72)=61.68 ; p<0.01 ; \mathrm{F}_{\text {genotype }}(1,18)=1.17 ; p=0.29\right.$; $\left.\mathrm{F}_{\text {genotype } \times \text { delay }}(4,72)=2.33 ; p=0.06\right)$ (Fig. 3 ).

In lactating females, large reward preference decreased across days $\left(F_{\text {delay }}(7,126)=22.09 ; p<0.01\right)$. Overall, the preference for the large reward was higher in AVP-deficient animals compared to control animals $\left(\mathrm{F}_{\text {genotype }}(1,18)=5.13 ; p=0.03\right)$, but no significant interactions between genotype and delay were observed $\left(\mathrm{F}_{\text {genotype }} \times\right.$ delay $(7,126)=$ $1.02 ; p=0.42$ ) (Fig. 4a). The number of inadequate responses was significantly increased throughout the test phase $\left(\mathrm{F}_{\text {delay }}(7,126)=\right.$ $11.67 ; p<0.01)$, but it was unaltered by genotype $\left(F_{\text {genotype }}(1,18)=\right.$ 3.7; $p=0.07)$. A significant interaction between genotype and delay was observed $\left(F_{\text {genotype }} \times\right.$ delay $\left.(7,126)=6.2 ; p<0.01\right)$, as post-hoc comparisons revealed, the increase in the number of inadequate responses was greater in control animals than in AVP-deficient rats (Fig. 4b).

Large reward preference was unchanged by genotype or treatment in lactating females during the three-day pharmacological treatment period $\left(F_{\text {genotype }}(1,18)=1.68 ; p=0.21 ; F_{\text {treatment }}(2,36)=1.16 ; p=\right.$ 0.33 ) (Fig. 4c). A significant interaction between genotype and treatment was observed $\left(\mathrm{F}_{\text {genotype }} \times\right.$ treatment $\left.(2,36)=5.29 ; p<0.01\right)$. Posthoc analyses revealed that both CDP and IMI significantly decreased large reward preference only in AVP-deficient rats compared to vehicle treatment. The number of inadequate responses was decreased in AVP-deficient rats compared to control animals and increased by CDP treatment compared to vehicle treated subjects, while IMI caused no significant changes in this variable $\left(\mathrm{F}_{\text {genotype }}(1,18)=7.4 ; p=0.01\right.$; $\mathrm{F}_{\text {treatment }}(2,36)=3.7 ; p<0.03$ ) (Fig. $\left.4 \mathrm{~d}\right)$. No significant interaction was observed between genotype and treatment $\left(\mathrm{F}_{\text {genotype }} \times\right.$ treatment $(2,36)=$ $2.76 ; p=0.08$ ).

HPA axis activity in AVP-deficient and control virgin and lactating female rats

AVP deficiency did not alter corticosterone levels 10 days after delivery in lactating or virgin rats $\left(\mathrm{F}_{\text {genotype }}(1,34)=0.15 ; p=0.7\right.$;
Virgin females

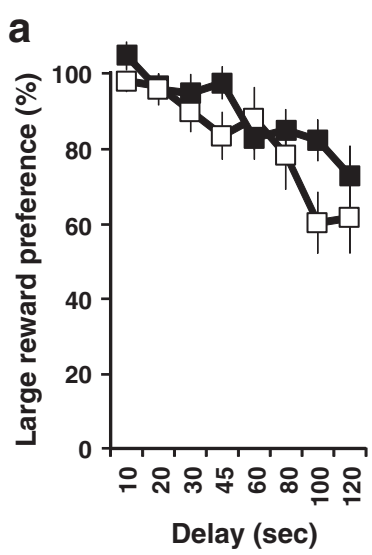

b

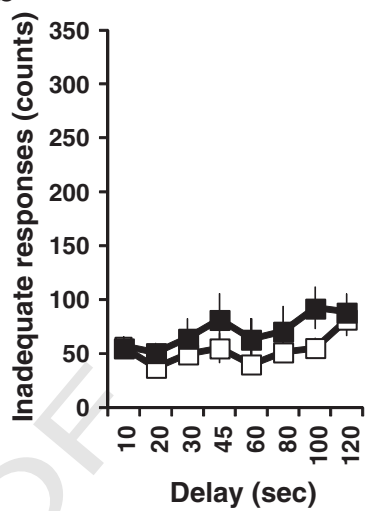

C

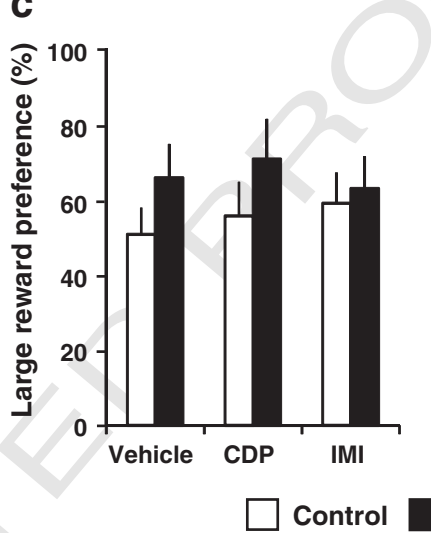

d

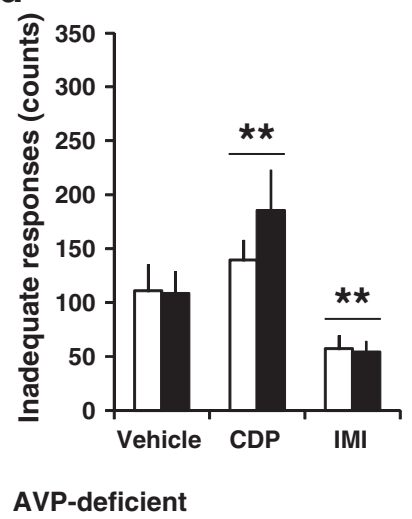

Fig. 2. Effects of vasopressin (AVP) deficiency on impulsivity in virgin female Brattleboro rats in the test phase of the delay discounting paradigm (Experiment 1). Control and AVPdeficient rats showed no differences in impulsivity $(\mathrm{a}-\mathrm{b})$. Chlordiazepoxide (CDP)-induced enhancement of GABAergic and imipramine (IMI)-induced enhancement of serotonergic activity resulted in no changes in large reward preference, an indicator of choiceimpulsivity (c). In both control and AVP-deficient virgins, CDP-treatment caused an increase, while IMI-treatment caused a decrease in the number of inadequate responses, an indicator of motor impulsivity (d). ${ }^{* *}$ above the line denotes a significant difference from control treatment group in post-hoc comparison $(p<0.01)$.

\section{Lactating females}

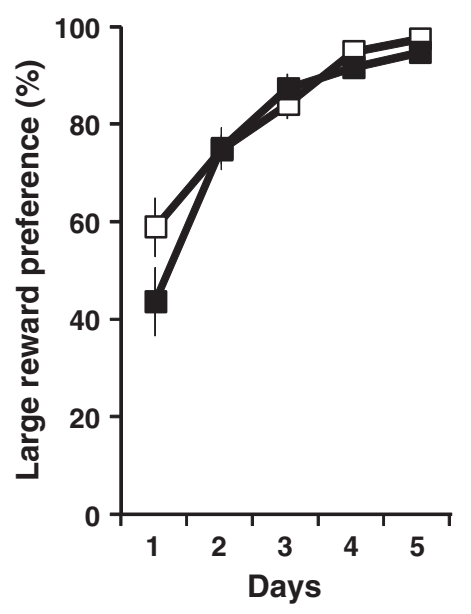

\section{Control AVP-deficient}

Fig. 3. Effects of vasopressin (AVP) deficiency on learning in lactating female Brattleboro rats in the training phase of the delay discounting paradigm (Experiment 2). AVP deficiency had no significant effects on learning ability. 


\section{Lactating females}

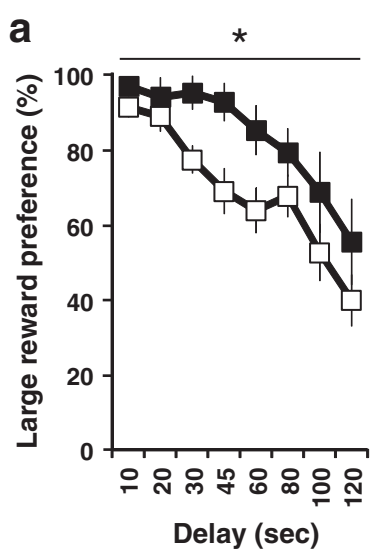

b

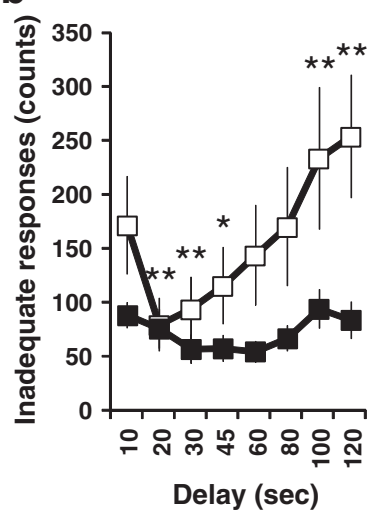

C

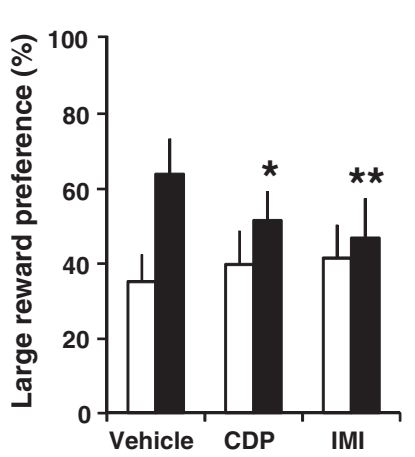

d

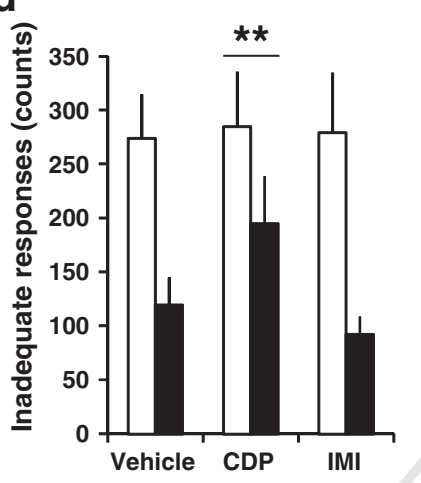

$\square$ Control $\square$ AVP-deficient

Fig. 4. Effects of vasopressin (AVP) deficiency on impulsivity in lactating female Brattleboro rats in the test phase of the delay discounting paradigm (Experiment 2). AVP-deficient rats showed decreased impulsivity (a-b). Both chlordiazepoxide (CDP)-induced enhancement of GABAergic activity and imipramine (IMI)-induced enhancement of serotonergic activity caused a decrease in large reward preference, an indicator of choice-impulsivity, but only in AVP-deficient rats (c). CDP-treatment caused an increase in the number of inadequate responses, an indicator of motor impulsivity in all rats regardless of their genotypes, while IMItreatment had no effect on the same variable (d). In Fig. 4a * over the line denotes an overall significant difference between the two genotypes in post-hoc comparison $(p<0.05)$; in Fig. $4 \mathrm{~b} *$ denotes a significant difference from value at 10 second delay $(p<0.05) ;{ }^{* *}$ denotes a significant difference from value at 10 second delay $(p<0.01)$; in Fig. $4 c *$ denotes a significant difference from control treatment group within the same genotype in post-hoc comparison $(p<0.05),{ }^{* *}$ denotes a significant difference from control treatment group within the same genotype in post-hoc comparison $(p<0.01)$; in Fig. $4 \mathrm{~d}^{* *}$ over the line denotes a significant difference from control treatment group in post-hoc comparison $(p<0.01)$.

$F_{\text {lactation }}(1,34)=1.23 ; p=0.27 ; F_{\text {genotype } \times \text { lactation }}(1,34)=2.82 ; p=$ 0.1 ) (Fig. 5a).

Corticosterone was unchanged by genotype or lactation $\left(\mathrm{F}_{\text {genotype }}(1,58)=2.95 ; p=0.09 ; \mathrm{F}_{\text {lactation }}(1,58)=3.52 ; p=0.06\right)$ when measured 20 days after delivery. A significant interaction was observed between AVP deficiency and lactation, as corticosterone levels were higher in control lactating than control virgin rats, but they did not differ in AVP-deficient lactating and virgin rats $\left(F_{\text {genotype }} \times\right.$ lactation $\left.(1,58)=5.86 ; p=0.01\right)($ Fig. $5 b)$.

\section{Discussion}

AVP-deficient virgin females exhibited decreased learning abilities when compared to control animals. However, AVP deficiency did not appear to affect impulsive behavior. CDP increased impulsivity and IMI decreased impulsivity in both genotypes of virgin females. Corticosterone levels were unaltered by AVP deficiency. In lactating females, AVP deficiency did not alter learning, but led to decreased impulsivity.

10th

20th

Day after delivery

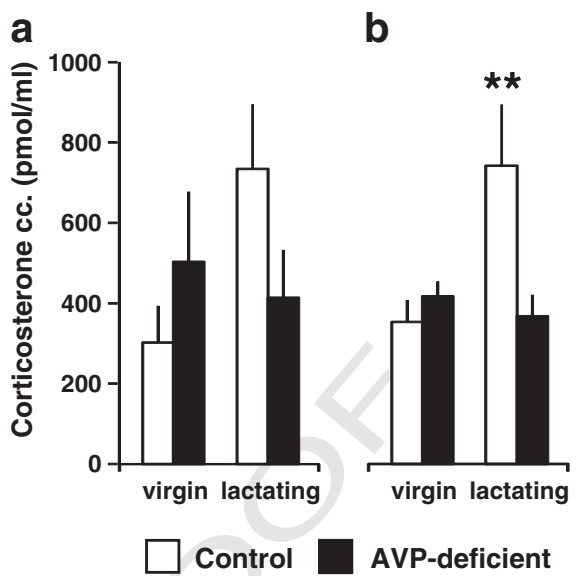

Fig. 5. Effects of vasopressin (AVP) deficiency on corticosterone levels in control and AVPdeficient virgin and lactating females 10 days (a) and 20 days (b) after delivery. AVPdeficiency abolished lactation-induced increases in corticosterone levels on the 20th day after delivery. ${ }^{* *}$ denotes a significant difference from the virgin group within the same genotype in post-hoc comparison $(p<0.01)$.

Both CDP and IMI decreased choice impulsivity, but only in AVP- 372 deficient rats. CDP increased motor impulsivity in both genotypes of 373 lactating rats. Lactating rats exhibited elevated corticosterone levels 374 at the end of the test phase of the delayed discounting test, but AVP 375 deficiency dampened this elevation.

376

Our results demonstrating that reduced learning capabilities in AVP- 377 deficient rats are in line with earlier reports in which Brattleboro 378 rats were shown to exhibit impaired performance in cognitive tasks 379 (Aarde and Jentsch, 2006; Colombo et al., 1992; Varga et al., 2013). In- 380 terestingly, lactation seems to alter these effects, as these effects of 381 AVP deficiency did not occur in lactating rats. Taken together, these 382 findings suggest that AVP plays a role in the modulation of cognitive 383 processes and that the specific physiological state during lactation de- 384 creases the sensitivity of animals to the effects of AVP deficiency. $\quad 385$

The interaction between AVP and lactation in the regulation of 386 impulsivity appears to be different than what is observed for cognitive 387 capabilities. The lack of AVP-induced effects on impulsive behavior in 388 virgin rats suggests that AVP does not modulate impulsivity under 389 non-lactating conditions. In contrast, AVP-deficient lactating rats exhib- 390 it decreased impulsivity, which might indicate that the neuroendocrine 391 state developing during lactation involves AVP in the regulation of im- 392 pulsivity. Similar lactation-dependent effects of AVP on impulsivity 393 have not been reported previously, but our findings are consistent 394 with studies showing that the behavioral effects of AVP deficiency 395 (e.g. reduced depressive-like behavior) occur in a lactation-dependent 396 manner in rats (Fodor et al., 2012).

An interesting trend was observed when comparing the number of 398 inadequate responses in the delay discounting test between virgin and 399 lactating females. While the number of inadequate responses showed 400 a small, AVP-independent increase throughout the test phase in virgin 401 rats, the number of inadequate responses increased remarkably with 402 the length of the delays in control, AVP-expressing lactating females. In- 403 terestingly, in AVP-deficient lactating subjects, the changes in inade- 404 quate responses were similar to those observed in virgin animals (see 405 Figs. 2b and 4b). These differences suggest that AVP does not play a 406 role in the regulation of impulsivity in virgin animals, but that it exerts 407 a robust, impulsivity-increasing effect in lactating rats. In the absence 408 of AVP, lactating female rats exhibit a less impulsive phenotype that is 409 similar to that observed in virgin female rats. Because the AVP system 410 is well known to be over-activated during lactation (Caldwell et al., 411 
1987; Landgraf et al., 1991) we can assume that the enhanced AVP activity in lactating rats might be one of the causes of their increased impulsivity. One might hypothesize that moderately increased impulsivity might be beneficial to lactating rats given that rapidly accessible sources would be preferred when energy need is constantly high (Widdowson, 1976).

AVP-deficiency exerted a lactation-dependent impact on the effects of pharmacological manipulations on impulsive behavior. In virgin rats, CDP treatment increased, while IMI treatment decreased impulsivity in a genotype-independent manner, which findings are in line with earlier data (Bizot et al., 1988; Evenden and Ko, 2005; Miyazaki et al., 2011; Thiebot et al., 1985; Wolff and Leander, 2002). In contrast, AVP deficiency altered the effects of pharmacological manipulations of GABAergic and serotonergic signaling on impulsivity during lactation: both treatments increased choice impulsivity in AVP-deficient lactating rats. As this form of impulsivity was unaltered by treatments in virgin AVPdeficient rats, one might assume that AVP only interacts with GABAergic and serotonergic signaling in the regulation of choice impulsivity during lactation. Interestingly, IMI treatment led to an increase in this form of impulsivity of AVP-deficient lactating rats in contrast to its motor impulsivity-decreasing effects in virgin animals; however, similar contrasting effects of serotonergic manipulations on different forms impulsivity had been reported earlier (Harrison et al., 1997; Winstanley et al., 2004). Although the precise effects of CDP and IMI on impulsivity have yet to be fully elucidated, our results demonstrate that AVP interacts with serotonergic and GABAergic signaling similarly in the regulation of choice impulsivity during lactation. Specifically, AVP desensitizes lactating rats to the choice impulsivity-altering effects of GABAergic and serotonergic manipulations, while AVP-deficient lactating rats react to such manipulations. Regarding motor impulsivity, AVP deficiency had no similar impact on the effects of pharmacological treatments, suggesting that interactions between AVP, GABAergic and serotoninergic signaling might be only involved in the regulation of choice impulsivity.

Corticosterone levels were increased in control lactating rats twenty days after delivery, while AVP deficiency dampened this increase, a finding that is consistent with earlier reports (Fodor et al., 2013).These data might suggest that AVP contributes, in part, to maternal increases in impulsivity via the enhancement of HPA axis activity, as earlier reports showed that chronic corticosterone treatment leads to increased impulsivity (Torregrossa et al., 2012).

\section{Conclusions}

Taken together, our results demonstrate that AVP is crucial for normal cognitive processes in virgin rats, but it does not exert similar effects during lactation. In contrast, AVP alters impulsive behavior in the opposite manner. Namely, it does not play a role in the regulation of impulsive behavior in virgin females, but it has an important impulsivityincreasing effect during lactation, possibly by increasing HPA axis activity. Moreover, AVP apparently desensitizes lactating females to the effects of GABAergic and serotonergic manipulations on choice impulsivity. These results contribute to our knowledge regarding the effects of AVP on cognitive processes and impulsivity, as well as the dependence of these effects on neuroendocrine background. Our findings highlight possible interactions between the AVPergic, GABAergic, serotonergic systems and the HPA axis, which might contribute to the development of maternal impulsive behavior.

\section{References}

Aarde, S.M., Jentsch, J.D., 2006. Haploinsufficiency of the arginine-vasopressin gene is associated with poor spatial working memory performance in rats. Horm. Behav. 49, 501-508.

Adriani, W., Laviola, G., 2003. Elevated levels of impulsivity and reduced place conditioning with d-amphetamine: two behavioral features of adolescence in mice. Behav. Neurosci. 117, 695-703.
Adriani, W., Caprioli, A., Granstrem, O., Carli, M., Laviola, G., 2003a. The spontaneously 474 hypertensive-rat as an animal model of ADHD: evidence for impulsive and non- 475 impulsive subpopulations. Neurosci. Biobehav. Rev. 27, 639-651.

Adriani, W.,Seta, D.D.,Dessi-Fulgheri, F.,Farabollini, F.,Laviola, G., 2003b. Altered profiles of 477 spontaneous novelty seeking, impulsive behavior, and response to D-amphetamine 478 in rats perinatally exposed to bisphenol A. Environ. Health Perspect. 111, 395-401. 479

Antoni, F.A., 1993. Vasopressinergic control of pituitary adrenocorticotropin secretion 480 comes of age. Front. Neuroendocrinol. 14, 76-122.

Aron, A.R. 2007. The neural basis of inhibition in 214-228.

Auerbach, S., Lipton, P., 1982. Vasopressin augments depolarization-induced release and 484

Bizot, J.C., Thiebot, M.H., 1996. Impulsivity as a confounding factor in certain animal tests 486 of cognitive function. Brain Res. Cogn. Brain Res. 3, 243-250.

Bizot, J.C. Thiebot, M.H. Le Bihan, C. Soubrie, P.Simon, P. 1988. Effects of imipramine-like 488 drugs and serotonin uptake blockers on delay of reward in rats. Possible implication 489 in the behavioral mechanism of action of antidepressants. J. Pharmacol. Exp. Ther. 490 246, 1144-1151.

Bizot, J. Le Bihan, C. Puech, AJ, Hamon, M. Thiebot, M., 1999. Serotonin and tolerance to delay of reward in rats. Psychopharmacology (Berlin) 146, 400-412.

Bohus, B.,de Wied, D., 1998. The vasopressin deficient Brattleboro rats: a natural knockout 494 model used in the search for CNS effects of vasopressin. Prog. Brain Res. 119, 555-573. 495

Buijs, R.M.,Swaab, D.F.,Dogterom, J.,van Leeuwen, F.W., 1978. Intra- and extrahypothalamic 496 vasopressin and oxytocin pathways in the rat. Cell Tissue Res. 186, 423-433. 497

Caldwell, J.D., Greer, E.R., Johnson, M.F., Prange Jr., A.J., Pedersen, C.A., 1987. Oxytocin and 498 vasopressin immunoreactivity in hypothalamic and extrahypothalamic sites in late 499 pregnant and postpartum rats. Neuroendocrinology 46, 39-47. 500

Colombo, G., Hansen, C., Hoffman, P.L., Grant, K.A., 1992. Decreased performance in a de- 501 layed alternation task by rats genetically deficient in vasopressin. Physiol. Behav. 502 52, 827-830. 503

Engelmann, M., Landgraf, R., 1994. Microdialysis administration of vasopressin into the 504 septum improves social recognition in Brattleboro rats. Physiol. Behav. 55, 145-149. 505

Evenden, J.L., 1998. The pharmacology of impulsive behaviour in rats III: the effects of 506 amphetamine, haloperidol, imipramine, chlordiazepoxide and ethanol on a paced 507 fixed consecutive number schedule. Psychopharmacology (Berlin) 138, 295-304. 508

Evenden, J.,Ko, T., 2005. The psychopharmacology of impulsive behaviour in rats VIII: effects 509 of amphetamine, methylphenidate, and other drugs on responding maintained by a 510 fixed consecutive number avoidance schedule. Psychopharmacology (Berlin) 180, 511 294-305.

Evenden, J.L., Ryan, C.N., 1996. The pharmacology of impulsive behaviour in rats: the 513 effects of drugs on response choice with varying delays of reinforcement. Psycho- 514 pharmacology (Berlin) 128, 161-170.

Evenden, J.L., Ryan, C.N., 1999. The pharmacology of impulsive behaviour in rats VI: the 516 effects of ethanol and selective serotonergic drugs on response choice with varying 517 delays of reinforcement. Psychopharmacology (Berlin) 146, 413-421. 518

Feifel, D., Mexal, S., Melendez, G., Liu, P.Y., Goldenberg, J.R., Shilling, P.D., 2009. The 519 brattleboro rat displays a natural deficit in social discrimination that is restored by 520 clozapine and a neurotensin analog. Neuropsychopharmacology 34, 2011-2018. 521

Flamion, B., Spring, K.R., 1990. Water permeability of apical and basolateral cell 522 membranes of rat inner medullary collecting duct. Am. J. Physiol. 259, F986-F999. 523

Fodor, A., Klausz, B., Pinter, O., Daviu, N., Rabasa, C., Rotllant, D., Balazsfi, D., Kovacs, K.B., 524 Nadal, R.,Zelena, D., 2012. Maternal neglect with reduced depressive-like behavior 525 and blunted c-fos activation in Brattleboro mothers, the role of central vasopressin. 526 Horm. Behav. 62, 539-551.

Fodor, A., Pinter, O., Domokos, A., Langnaese, K., Barna, I., Engelmann, M.,Zelena, D., 2013. 528 Blunted HPA axis response in lactating, vasopressin-deficient Brattleboro rats. 529 J. Endocrinol. 219, 89-100.

Harrison, A.A.,Everitt, B.J., Robbins, T.W., 1997. Central 5-HT depletion enhances impulsive 531 responding without affecting the accuracy of attentional performance: interactions 532 with dopaminergic mechanisms. Psychopharmacology (Berlin) 133, 329-342. 533

Hermes, M.L., Ruijter, J.M., Klop, A., Buijs, R.M., Renaud, L.P., 2000. Vasopressin increases 534 GABAergic inhibition of rat hypothalamic paraventricular nucleus neurons in vitro. 535 J. Neurophysiol. 83, 705-711.

Kim, S.,Lee, D., 2011. Prefrontal cortex and impulsive decision making. Biol. Psychiatry 69, 537 1140-1146.

ndgraf, R., Neumann, I.,Pittman, Q.J., 1991. Septal and hippocampal release of vasopres- 539 sin and oxytocin during late pregnancy and parturition in the rat. Neuroendocrinolo- 540 gy $54,378-383$. kara, G.B., Varga, J., Barna, I., Pinter, O., Klausz, B., Zelena, D., 2012. The vasopressin- 542 deficient Brattleboro rat: lessons for the hypothalamo-pituitary-adrenal axis regula- 543 tion. Cell. Mol. Neurobiol. 32, 759-766.

Miyazaki, K., Miyazaki, K.W., Doya, K., 2011. Activation of dorsal raphe serotonin neurons 545 underlies waiting for delayed rewards. J. Neurosci. 31, 469-479. 546

Mlynarik, M., Zelena, D., Bagdy, G., Makara, G.B., Jezova, D., 2007. Signs of attenuated 547 depression-like behavior in vasopressin deficient Brattleboro rats. Horm. Behav. 51, 548 395-405.

Panlilio, L.V.,Justinova, Z., Mascia, P., Pistis, M., Luchicchi, A., Lecca, S., Barnes, C., Redhi, G.H., 550 Adair, J.,Heishman, S.J.,Yasar, S.,Aliczki, M.,Haller, J., Goldberg, S.R., 2012. Novel use of 551 a lipid-lowering fibrate medication to prevent nicotine reward and relapse: preclini- 552 cal findings. Neuropsychopharmacology 37, 1838-1847.

Ramanathan, G., Cilz, N.I., Kurada, L., Hu, B., Wang, X., Lei, S., 2012. Vasopressin facilitates 554 GABAergic transmission in rat hippocampus via activation of $V(1 \mathrm{~A})$ receptors. Neuro- 555 pharmacology 63, 1218-1226.

Rhodes, C.H., Morrell, J.I.,Pfaff, D.W., 1981. Immunohistochemical analysis of magnocellular 557 elements in rat hypothalamus: distribution and numbers of cells containing 558 neurophysin, oxytocin, and vasopressin. J. Comp. Neurol. 198, 45-64. 
Schank, J.C., 2009. Early locomotor and social effects in vasopressin deficient neonatal rats. Behav. Brain Res. 197, 166-177.

Schwarzberg, H., Kovacs, G.L., Szabo, G., Telegdy, G., 1981. Intraventricular administration of vasopressin and oxytocin effects the steady-state levels of serotonin, dopamine and norepinephrine in rat brain. Endocrinol. Exp. 15, 75-80.

Sokol, H.W.,Zimmerman, E.A.,Sawyer, W.H., Robinson, A.G., 1976. The hypothalamicneurohypophysial system of the rat: localization and quantitation of neurophysin by light microscopic immunocytochemistry in normal rats and in Brattleboro rats deficient in vasopressin and a neurophysin. Endocrinology 98, 1176-1188.

Solanto, M.V.,Abikoff, H.,Sonuga-Barke, E.,Schachar, R.,Logan, G.D.,Wigal, T.,Hechtman, L., Hinshaw, S.,Turkel, E., 2001. The ecological validity of delay aversion and response inhibition as measures of impulsivity in $\mathrm{AD} / \mathrm{HD}$ : a supplement to the NIMH multimodal treatment study of AD/HD. J. Abnorm. Child Psychol. 29, 215-228.

Thiebot, M.H., Le Bihan, C., Soubrie, P., Simon, P., 1985. Benzodiazepines reduce the tolerance to reward delay in rats. Psychopharmacology (Berlin) 86, 147-152

Torregrossa, M.M.,Xie, M., Taylor, J.R., 2012. Chronic corticosterone exposure during adolescence reduces impulsive action but increases impulsive choice and sensitivity to yohimbine in male Sprague-Dawley rats. Neuropsychopharmacology $37,1656-1670$.

Varga, J.,Klausz, B.,Domokos, A., Kalman, S.,Pakaski, M.,Szucs, S., Garab, D.,Zvara, A.,Puskas, L., Kalman, J., Timar, J., Bagdy, G., Zelena, D., 2013. Increase in Alzheimer's related markers preceeds memory disturbances: studies in vasopressin-deficient Brattleboro 581 rat. Brain Res. Bull. 100C, 6-13.

Wade, J.B., Stetson, D.L., Lewis, S.A., 1981. ADH action: evidence for a membrane shuttle 583 mechanism. Ann. N. Y. Acad. Sci. 372, 106-117.

Wang, J.,Irnaten, M.,Venkatesan, P.,Evans, C., Mendelowitz, D., 2002. Arginine vasopressin 585 enhances GABAergic inhibition of cardiac parasympathetic neurons in the nucleus 586 ambiguus. Neuroscience 111, 699-705.

Widdowson, EM 1976. Changes in the body and its organs during lactation: nutritional 588 implications. Ciba Found. Symp. 103-118.

Winstanley, C.A., Dalley, J.W., Theobald, D.E., Robbins, T.W., 2004. Fractionating impulsivi- 590 ty: contrasting effects of central 5-HT depletion on different measures of impulsive 591 behavior. Neuropsychopharmacology 29, 1331-1343.

Wolff, M.C., Leander, J.D., 2002. Selective serotonin reuptake inhibitors decrease 593 impulsive behavior as measured by an adjusting delay procedure in the pigeon. 594 Neuropsychopharmacology 27, 421-429.

Zelena, D.,Mergl, Z., Foldes, A.,Kovacs, K.J.,Toth, Z., Makara, G.B., 2003. Role of hypothalam- 596 ic inputs in maintaining pituitary-adrenal responsiveness in repeated restraint. Am. 597 J. Physiol. Endocrinol. Metab. 285, E1110-E1117.

Zelena, D.,Domokos, A. Jain, S.K. Jankord, R.,Filaretova, L, 2009. The stimuli-specific role of vasopressin in the hypothalamus-pituitary-adrenal axis response to stress. 600 J. Endocrinol. 202, 263-278. 